UDC 502.175-047.36(470.45)

LBC 20.18(2P-4Вог)

\title{
ATOMIC ABSORPTION SPECTROMETRY IN LOCAL MONITORING OF HEAVY METALS (ON THE EXAMPLE OF SOLID SEDIMENTS)
}

\author{
Anna A. Tikhonova \\ Volgograd State University, Volgograd, Russian Federation
}

\begin{abstract}
The article considers features of the method of atomic absorption spectrometry and its variants. Special attention is paid to the relevance and peculiarities of its application in monitoring the content of heavy metals in different environments on a local and regional level. With the help of KVANT.Z atomic absorptive spectrometer (the company-producer is KORTEK) the author determines the actual volume of inputs of heavy metals from a specific local source. The metallurgical Krasnyy oktyabr plant was chosen as such a local source. The determination of the actual concentrations of heavy metals covered the winter period 2016-2017 and included measurement of content of individual elements in the freshly fallen solid precipitation. Sampling of solid precipitation was carried out in the band interval of 50-1200 meters from the impact of the object on the basis of a triangulated irregular network. The results of measurements, for example total content of zinc, indicate the possibility of determining the qualitative elemental composition of the studied environments, a reflection of the number concentration of the specific element at each point of sampling. The obtained data of local monitoring allows to evaluate effectively the actual area of a specific local object impact. This is especially urgent for the urban environment.
\end{abstract}

Key words: atomic absorption spectrometry, heavymetals, local monitoring, environmental monitoring, steel industry.

УДК 502.175-047.36(470.45)

ББК 20.18(2Р-4Вог)

\section{АТОМНО-АБСОРБЦИОННАЯ СПЕКТРОМЕТРИЯ В ЛОКАЛЬНОМ МОНИТОРИНГЕ ТЯЖЕЛЫХ МЕТАЛЛОВ (НА ПРИМЕРЕ ТВЕРДЫХ ОСАДКОВ)}

\author{
Анна Афанасьевна Тихонова \\ Волгоградский государственный университет, г. Волгоград, Российская Федерация
}

\begin{abstract}
Аннотация. В работе рассмотрены метод атомно-абсорбционной спектрометрии и его разновидности, актуальность и особенности применения при проведении мониторинга содержания тяжелых металлов в различных средах на локальном и региональном уровнях. С помощью атомно-абсорбционного спектрометра «KBAHT.Z» (производство ОOO «КОРТЕК») проводилось определение фактических объемов поступления тяжелых металлов от конкретного локального источника в зимний период 2016-2017 гг. посредством измерения содержания отдельных элементов в свежевыпавших твердых осадках. В качестве объекта воздей두의 стия окружающую среду был выбран ВМК «Красный Октябрь» г. Волгограда. Отбор проб проводился в интервале 50-1200 м от объекта воздействия. Представленные результаты измерений на примере валового содержания цинка указывают на возможность определения качественного элементного состава исследуемых сред с отражением количества концентрации конкретного элемента в каждой точке отбора проб и, следовательно, позволяют оценить фактическую зону влияния конкретного локального объекта, что является весьма актуальным для определения качества городской среды.

Ключевые слова: атомно-абсорбционная спектрометрия, тяжелые металлы, локальный мониторинг, экологический мониторинг, черная металлургия.
\end{abstract}


Современное антропогенное воздействие на окружающую природную среду затрагивает все ее компоненты: атмосферный воздух, поверхностные и подземные воды, почвенный покров и т. д. В частности, негативное воздействие на почву заключается в нарушении ее структуры и привнесении новых чужеродных веществ, приводящем к изменению химического состава и снижению уровня благоприятности для живых организмов [23]. Для городских территорий в связи с комплексным воздействием большого количества разного вида источников (таких как промышленные предприятия, автомобильный и железнодорожный транспорт, селитебные зоны и др.) проблема загрязнения почвенного покрова как основной депонирующей среды тяжелыми металлами и иными токсичными элементами особенно актуальна [22].

Однако, помимо мониторинга непосредственно состояния почв, необходим контроль не только за источниками привнесения загрязняющих веществ, но и за средами и путями, по которым данные вещества попадают в почвенный покров, включая объем фактического поступления и характер перемещения между средами.

Ежегодные мониторинговые исследования в целях контроля за содержанием тяжелых металлов в почвах всех категорий осуществляются местными органами исполнительной власти в области охраны окружающей среды. По г. Волгограду их проводит Комитет природных ресурсов и экологии Волгоградской области на следующих территориях $[2 ; 3]$ :

- особо охраняемая природная территория муниципального значения (пойма р. Царицы);

- территории, подвергающиеся наибольшим техногенным нагрузкам (полигоны ТБО Тракторозаводского, Кировского, Красноармейского районов);

- территории, прилегающие к водным объектам, находящимся в муниципальной собственности г. Волгограда.

Результаты мониторинговых обследований почвы показывают, что основная масса загрязняющих веществ аккумулируется вблизи промышленных предприятий (поскольку каждый локальный источник характеризуется своей зоной влияния и ареалом рассеяния загрязняющих веществ, в частности тяжелых металлов), тем самым вызывая локальное загрязнение территории. Максимальным же загрязнениям подвержены почвы на территориях, расположенных вдоль крупных автодорог $[4 ; 21]$.

На сегодняшний день одним из наиболее приоритетных методов определения содержания тяжелых металлов в различных средах является метод атомно-абсорбционной спектрометрии, основанный на измерении поглощения резонансного излучения свободными атомами, находящимися в газовой фазе, за относительно короткое время [20].

При этом существует два принципиально отличных вида ионизирующего излучения: линейный и непрерывный. Соответственно имеют место различные методы перевода определяемого вещества в атомный пар (виды атомизаторов) [16]. При линейном излучении к ним относятся пламенный, электротермический (непламенный) и метод холодного пара. Атомизация с использованием источника непрерывного излучения (дейтериевые и галогеновые лампы) обеспечивает более широкий спектральный диапазон, а следовательно, более высокую разрешающую способность по сравнению с применением источников линейного излучения $[17 ; 18]$.

Все вышеперечисленное обусловливает как высокую степень точности результатов, так и широту спектра определяемых элементов. Оборудование, позволяющее реализовывать подобные исследования, представлено различными моделями спектрометров: contrAA800 series, SavantAA, SensAA, AA6800, novAA400 P, ZEEnit 650 Р и др. [1; 19; 22]

Цель исследования - определение целесообразности и обоснованности применения метода атомно-абсорбционной спектрометрии при решении задач локального экологического мониторинга тяжелых металлов, в частности определении фактического их поступления в среды и перемещения по геохимическим потокам, с учетом современной лабораторной базы.

\section{Методы исследования}

В качестве исследуемого локального объекта воздействия на окружающую среду был выбран ВМК «Красный Октябрь» г. Вол- 


\section{ЭКОЛОГИЯ И ПРИРОДОПОЛЬЗОВАНИЕ}

гограда. Данное предприятие в настоящее время - один из крупнейших производителей качественного металлопроката специальных марок стали для предприятий автомобилестроения и авиационной промышленности, химического, нефтяного и энергетического машиностроения, нефтегазодобывающей промышленности в РФ [11] и, следовательно, является крупным источником привнесения поллютантов (в том числе тяжелых металлов) в окружающую среду.

Пробоотбор свежевыпавших твердых осадков (снега) проводился по установленной методике [7] с подветренной стороны в зоне 50-1200 м, включая санитарно-защитную зону указанного промышленного предприятия.

Пробоподготовка, согласно методике [5], включала в себя перевод осадков в жидкую фазу и отстаивание в течение суток для осаждения механических примесей. Анализ производился в 10-дневный срок от даты забора проб с помощью атомно-абсорбционного спектрометра «KBAHT.Z» (производство ООО «КОРТЕК») [5; 13], характеризующегося универсальностью, востребованностью и доступностью вкупе с сохранением достаточного для проводимых исследований уровня точности.

Основной функцией указанного спектрометра является определение концентрации множества элементов в жидких пробах различного происхождения и состава $[13 ; 21]$. Характеристики прибора позволяют определять содержание элементов в почвах, атмосферных осадках, растительности, нефтепродуктах, сточных водах, ПАВ, продуктах питания, удобрениях и т. д., что соответствует задачам прикладных и мониторинговых исследований локального и регионального уровней.

Принцип работы спектрометра основан на анализе степени селективного поглощения резонансных спектральных линий атомным паром определяемого элемента [13; 24]. При этом для каждого элемента существует собственная аналитическая резонансная линия, обеспечивающая максимальное поглощение света, источником которого служат лампы с полным катодом для каждого определяемого элемента (линейное излучение).

Процесс атомизации (перевода анализируемой пробы в атомный пар) производится в аналитической ячейке электротермического атомизатора. Нагрев графитовой печи до температуры, необходимой для испарения пробы и атомизации элемента, осуществляется электрическим током [15].

В связи с наличием в аналитической ячейке, помимо атомов определяемого элемента, газообразных компонентов, способных поглощать падающий свет, имеет место явление неатомного, или фонового, поглощения, которое, в свою очередь, является источником систематической погрешности измерений. Для автоматической коррекции данного типа поглощения в спектрометре используется обратный эффект Зеемана, обеспечиваемый размещением графитовой печи в продольном переменном магнитном поле [15].

Поскольку прибор позволяет определять концентрации широкого спектра элементов различного происхождения, при проведении анализа применяются соответствующие объекту исследования методики [6; 8-10; 12], которые определяют порядок пробоподготовки и выполнения измерений для конкретного анализируемого образца [7; 14].

\section{Калибровка и проведение измерений}

Выполнение измерений на спектрометре осуществляется в несколько этапов. Первый включает в себя загрузку уже имеющейся или создание новой калибровки для анализируемого элемента.

Создание новой калибровки происходит в несколько этапов [13]:

1) в главном окне программы «КBAHT.Z» открывается методика на определяемый элемент, в пункте «Калибровки» выбирается опция «Создать»;

2) в появившемся окне «Создание калибровки» устанавливается тип аналитического сигнала («Пик» либо «Интеграл»), в пункте «Размерность» - единица концентрационной размерности, в пункте «Объем» - значение объема аликвоты, далее заполняются окна «Концентрация», «Название», «Описание»;

3) поочередно измеряются фоновые растворы (стандарты) с заданной концентрацией калибруемого элемента (с отклонением до $2 \%$ ), причем количество параллельных измерений (не менее двух) каждого раствора зара- 


\section{Показатели содержания тяжелых металлов (на примере цинка) в пробах снега вблизи ВМК «Красный Октябрь» (по состоянию на 06.02.2017 г.)}

\begin{tabular}{|l|c|c|c|}
\hline Образец & $\begin{array}{c}\text { Удаленность } \\
\text { от источника, м }\end{array}$ & $\begin{array}{c}\text { Средняя } \\
\text { концентрация, мкг/л }\end{array}$ & $\begin{array}{c}\text { Среднеквадратическое } \\
\text { отклонение }\end{array}$ \\
\hline Проба 1 & 250 & 27,419 & 1,257 \\
\hline Проба 2 & 500 & 12,483 & 0,735 \\
\hline Проба 3 & 50 & 29,405 & 2,153 \\
\hline Проба 4 & 300 & 20,574 & 1,685 \\
\hline Проба 5 & 550 & 35,757 & 2,495 \\
\hline Проба 6 & 200 & 9,453 & 0,925 \\
\hline Проба 7 & 450 & 19,960 & 2,416 \\
\hline Проба 8 & 700 & 75,175 & 2,450 \\
\hline Проба 9 & 1200 & 25,044 & 1,870 \\
\hline Проба 10 & 200 & 13,250 & 1,370 \\
\hline Проба 11 & 450 & 37,523 & 1,938 \\
\hline Проба 12 & 200 & 25,341 & 1,921 \\
\hline
\end{tabular}

Примечание. Сост. авт.

нее не устанавливается, а определяется статистическими характеристиками серии;

4) после окончания измерений последнего стандарта в окне «Создание калибровки» будут отражены все калибровочные точки и аппроксимирующая кривая. На данном этапе осуществляется выбор типа кривой (линейная, параболическая либо дробно-рациональная), затем вновь созданная калибровка сохраняется.

На втором этапе осуществляются выбор нужной калибровки, настройка параметров методики и собственно измерение анализируемых проб. Для этого оператором открывается окно «Измерение», в пункте меню «Вид» выбираются метрологические и иные характеристики, которые будут отражены при проведении измерений. Затем в строку «Новый образец» вводится название анализируемого образца, и после нажатия кнопки «Добавить» оно появится в таблице измерений [13]. При наличии нескольких проб для единовременного анализа программа позволяет добавить сразу все названия образцов и проводить измерение в указанной последовательности либо добавлять новые строки непосредственно перед загрузкой пробы в печь.

Затем оператором с помощью настроенной микропипетки (дозатора) осуществляется дозирование аликвоты анализируемой пробы в графитовую печь. При нажатии кнопки «Измерение», расположенной в верхней строке окна «Измерения», запускается программа нагрева графитовой печи, и в правой части экрана по- является окно «Аналитический сигнал», в котором индицируется текущая стадия нагрева и еепротекание [13]. После окончания программы нагрева в вышеуказанном окне отображаются импульс атомной абсорбционности и его амплитудное значение и интеграл. При этом в таблице окна «Измерение» отражаются амплитудное значение сигнала и значение концентрации, рассчитанное по калибровочной зависимости. Начиная со второго измерения в таблицу результатов добавляются значения среднего квадратического и относительного среднего квадратического отклонений [13].

Завершающим этапом работы на спектрометре является формирование оперативного отчета, для чего в меню окна «Измерения» в пункте «Отчет» выбирается форма отчета «Текст» или «Таблица» и полученные результаты сохраняются отдельным файлом. Кроме того, результаты измерений могут быть сохранены в сводной таблице, где производится их обработка с учетом параметров предварительной подготовки анализируемой пробы. Для этого в окне «Измерения» необходимо нажать кнопку «Сохранить» [13].

Результаты и обсужндение. Полученные с помощью измерений данные позволяют судить о содержании анализируемых элементов в исследуемой среде на конкретной территории, в данном случае - в зоне влияния локального источника загрязнения окружающей среды (Волгоградский металлургический завод «Красный Октябрь»). Результаты анализа проб свежевыпавших твердых осадков 
на примере валового содержания цинка отражены в таблице.

Следует заметить, что представленные в таблице данные позволяют судить о фактическом содержании конкретного химического элемента в каждой отдельно взятой пробе. Так, на основе подобного анализа можно получить достоверную и актуальную информацию о качественном элементном составе исследуемой среды на какой-либо территории с отражением количества концентрации конкретного элемента в отдельных точках.

\section{Заключение}

Таким образом, при проведении мониторинга содержания тяжелых металлов в различных средах (таких как атмосферные осадки, почва, живые организмы) на локальном и региональном уровнях (что зависит от площади исследуемой территории и частоты точек пробоотбора) использование метода атомноабсорбционной спектрометрии и, в частности, атомно-абсорбционного спектрометра «KBAHT.Z» с электротермической атомизацией, позволяет выявить и оценить фактическую зону влияния конкретного локального объекта, что весьма актуально при определения качества городской среды.

\section{СПИСОК ЛИТЕРАТУРЫ}

1. Атомно-абсорбционный спектрометр AA6800 (Shimadzu) // Современная исследовательская инфраструктура Российской Федерации. Электрон. текстовые дан. - Режим доступа: http:// www.ckp-rf.ru/ckp/equipped/?SECTION_ID $=502 \&$ ELEMENT_ID=249854 (дата обращения: 17.02.2017).Загл. с экрана.

2. Доклад «О состоянии окружающей среды Волгоградской области в 2013 году» / редкол.: П. В. Вергун [и др.] ; М-во природ. ресурсов и экологии Волгогр. обл. - Волгоград : СМОТРИ, 2014. $300 \mathrm{c}$.

3. Доклад «О состоянии окружающей среды Волгоградской области в 2014 году» / редкол.: П. В. Вергун [и др.] ; Ком. природ. ресурсов и экологии Волгогр. обл. - Волгоград : СМОТРИ, 2015. $300 \mathrm{c}$.

4. Доклад «О состоянии окружающей среды Волгоградской области в 2015 году» / редкол.: В. Е. Сазонов [ и др.] ; Ком. природ. ресурсов и экологии Волгогр. обл. - Волгоград : СМОТРИ, 2016. - $300 \mathrm{c}$.

5. Другов, Ю. С. Пробоподготовка в экологическом анализе : практическое руководство / Ю. С. Другов, А. А. Родин. - 3-е изд., доп. и перераб. - М. : БИНОМ. Лаборатория знаний, 2015. - 855 с.

6. Методика выполнения измерений массовой доли металлов в почвах методами атомно-эмиссионной и атомно-абсорбционной спектрометрии ( $\mathrm{Mn}, \mathrm{Cu}$, As, Ni, Hg, Zn). M-МВИ-80-2001.-СПб., 2001.-30 c.

7. Методические рекомендации по проведению полевых и лабораторных исследований почв и растений при контроле загрязнения окружающей среды металлами / Ин-т эксперим. метеорологии, МГУ им. М. В. Ломоносова ; под ред. Н. Г. Зырина, С. Г. Малахова. - М. : Гидрометеоиздат. Моск. отд-ние, 1981. - 109 с.

8. Методические указания по выполнению измерений массовых концентраций алюминия, бария, бериллия, ванадия, висмута, железа, кадмия, калия, кальция, кобальта, кремния, лития, магния, марганца, меди, молибдена, мышьяка, натрия, никеля, олова, свинца, селена, серебра, стронция, сурьмы, таллия, теллура, титана, хрома, цинка в питьевых, природных и сточных водах на атомно-абсорбционном спектрометре «KBAHT.Z» с электротермической атомизацией. - М., 2010. - 8 с.

9. Методические указания по определению тяжелых металлов в почвах сельхозугодий и продукции растениеводства. - М. : Тайфун, 1992. $31 \mathrm{c}$.

10. МУК 4.1.1471-03. Атомно-абсорбционное определение массовой концентрации ртути в почвах и твердых минеральных материалах : (утв. Минздравом России 29.06.2003). - М., 2003. - 10 с.

11. О предприятии // ВМК «Красный Октябрь». - Электрон. текстовые дан. - Режим доступа: http://www.vmzko.ru/home/about.html (дата обращения: 15.02.2017). - Загл. с экрана.

12. РД 52.18.191-89. Методические указания. Методика выполнения измерений массовой доли кислоторастворимых форм металлов (меди, свинца, цинка, никеля, кадмия) в пробах почвы атомноабсорбционным анализом : (утв. Госкомгидрометом СССР). - М., 1990. -37 c.

13. Спектрометр атомно-абсорбционный с электротермической атомизацией «КВАHT.Z». Электрон. текстовые дан. - Режим доступа: http:// www.cortec.ru/index.php?id=30 (дата обращения: 15.02.2017). - Загл. с экрана.

14. Способы пробоподготовки почвы, донных отложений и твердых отходов для атомно-абсорбционного определения тяжелых металлов / В. И. Сафарова, Г. Ф. Шайдулина, Т. Н. Михеева [и др.] // Заводская лаборатория. Диагностика материалов. -2010 . - Т. 76, № 2. - С. 10-14. 
15. Atomabsorptionsspektrometer - Aufbau und virtuelles Messgerät // ChemgaPedia. - Electronic text data. - Mode of access: http://www.chemgapedia.de/ vsengine/vlu/vsc/de/ch/16/anac/aasvirtmess.vlu/ Page/vsc/de/ch/16/anac/aas5_unt1.vscml.html (date of access: 15.02.2017). - Title from screen.

16. Atomabsorptionsspektroskopie (AAS) Anwendung // ChemgaPedia 14.02.2017. - Electronic text data. - Mode of access: http://www.chemgapedia. de/vsengine/vlu/vsc/de/ch/16/ anac/aasanwend.vlu. html (date of access: 14.02.2017). - Title from screen.

17. Atomabsorptionsspektroskopie (AAS) und Atomemissionsspektroskopie (AES) // Johannes Gutenberg-Universität Mainz 16.02.2017. - Electronic text data. - Mode of access: http://www.ak-hoffmann. chemie.uni-mainz.de/pdf/script/AnalytC-Teil-4folien.pdf. - Title from screen.

18. Atomabsorptionsspektrometrie (AAS) // Universität Regensburg. - Electronic text data. Mode of access: http://www-app.uni-regensburg.de/ Fakultaeten/CHP/Analytische_Chemie/web/dateien/ duerkop/AAS.pdf(date of access: 16.02.2017). - Title from screen.

19. Atomic absorption spectrometers (AAS) // Green Lab Hungary Engineering, Ltd. 16.02.2017. Electronic text data. - Mode of access: http://www. greenlab.hu/atomic-absorption-spectrometersaas4.html?tid=zeenit-atomic-absorption-spectrometeraas. - Title from screen.

20. Einführung in die Atomabsorptionsspektroskopie (AAS) // ChemgaPedia. - Electronic text data. - Mode of access: http://www.chemgapedia.de/vsengine/vlu/ $\mathrm{vsc} / \mathrm{de} / \mathrm{ch} / 16 /$ anac/aaseinf.vlu.html (date of access: 14.02.2017). - Title from screen.

21. Klemm, K. Schnell! Einfach! Wirtschaftlich! Schnelle Multielement-Analyse von Schwermetallen in Boden- und Sedimentproben mit dem contrAA $\AA /$ K. Klemm, Dr. B. Özmen // Analytik Jena. 16.02.2017.Electronic text data. - Mode of access: https:// www.analytik-jena.de/en/analytical-instrumentation/ ssc/applicationsupport/downloads.html?tx _padb dam $\% 5$ Bpublication $\% 5 \mathrm{D}=\% 0 \mathrm{~A} \% \mathrm{EE} \% 02 \mathrm{y} \% \mathrm{FCM}$ $\%$ C6\%D8\&tx_padb_dam $\% 5$ Baction $\% 5 \mathrm{D}=$ download\&tx_padb_dam $\% 5$ Bcontroller $\% 5 \mathrm{D}=$ Download\&cHash=a3e4de429d82a36bb83c8b19e159 b633. - Title from screen.

22. Schwermetall-Emissionen // Das Umweltbundesamt. - Electronic text data. - Mode of access: http://www.umweltbundesamt.de/daten/ luftbelastung/luftschadstoff-emissionen-indeutschland/schwermetall-emissionen\#textpart-1 (date of access: 14.02.2017). - Title from screen.

23. Spangl, W. Jahresbericht der Luftgütemessungen in Österreich 2015 / W. Spangl, C. Nagl. - Wien : Umweltbundesamt GmbH, 2016. $152 \mathrm{~S}$.
24. Spektrallinien // ChemgaPedia. - Electronic text data. - Mode of access: http://www. chemgapedia. $\mathrm{de} /$ vsengine/vlu/vsc/de/ch/13/vlu/analytik/aas/ spektrallinien.vlu.html (date of access: 14.02.2017). Title from screen.

\section{REFERENCES}

1. Atomno-absorbtsionnyy spektrometr AA6800 (Shimadzu) [Atomic Absorption Spectrometer AA6800 (Shimadzu)]. Sovremennaya issledovatelskaya infrastruktura Rossiyskoy Federatsii [Modern Research Infrastructure of the Russian Federation]. URL: http://www.ckp-rf.ru/ckp/equipped/?SECTION $\mathrm{ID}=502 \& E L E M E N T$ ID $=249854$. (accessed February 17, 2017).

2. Vergun P.V., et al. Doklad «O sostoyanii okruzhayushchey sredy Volgogradskoy oblasti v 2013 godu» [The Report "On the State of the Environment of the Volgograd Region in 2013"]. Ministerstvo prirodnykh resursov i ekologii Volgogradskoy oblasti [Ministry of Natural Resources and Ecology of the Volgograd Region]. Volgograd, SMOTRI Publ., 2014. $300 \mathrm{p}$.

3. Vergun P.V., et al. Doklad «O sostoyanii okruzhayushchey sredy Volgogradskoy oblasti V 2014 godu» [The Report "On the State of the Environment of the Volgograd Region in 2014"]. Komitet prirodnykh resursov i ekologii Volgogradskoy oblasti [Committee of Natural Resources and Ecology of the Volgograd Region]. Volgograd, SMOTRI Publ., 2015. $300 \mathrm{p}$.

4. Sazonov V.E., et al. Doklad «O sostoyanii okruzhayushchey sredy Volgogradskoy oblasti V 2015 godu» [The Report "On the State of the Environment of the Volgograd Region in 2015"]. Komitet prirodnykh resursov i ekologii Volgogradskoy oblasti [Committee of Natural Resources and Ecology of the Volgograd Region]. Volgograd, SMOTRI Publ., 2016. $300 \mathrm{p}$.

5. Drugov Yu.S., Rodin A.A. Probopodgotovka $v$ ekologicheskom analize: prakticheskoe rukovodstvo [Sample Preparation in Ecological Analysis: Practical Guidance]. Moscow, BINOM. Laboratoriya znaniy Publ., 2015. 855 p.

6. Metodika vypolneniya izmereniy massovoy doli metallov $v$ pochvakh metodami atomnoemissionnoy $i$ atomno-absorbtsionnoy spektrometrii (Mn, Cu, As, Ni, Hg, Zn). M-MVI-80-2001 [Method for Performing Measurements of the Mass Fraction of Metals in Soils Using Atomic Emission and Atomic Absorption Spectrometry (Mn, Cu, As, Ni, Hg, Zn). M-MBI-80-2001]. Saint Petersburg, 2001.30 p.

7. Zyrin N.G., Malakhov S.G., eds. Metodicheskie rekomendatsii po provedeniyu polevykh $i$ 


\section{ЭКОЛОГИЯ И ПРИРОДОПОЛЬЗОВАНИЕ}

laboratornykh issledovaniy pochv $i$ rasteniy pri kontrole zagryazneniya okruzhayushchey sredy metallami [Methodological Recommendations for Conducting Field and Laboratory Studies of Soils and Plants in the Control of Environmental Pollution by Metals]. Moscow, Gidrometeoizdat. Mosk. otd-nie, $1981.109 \mathrm{p}$.

8. Metodicheskie ukazaniya po vypolneniyu izmereniy massovykh kontsentratsiy alyuminiya, bariya, berilliya, vanadiya, vismuta, zheleza, kadmiya, kaliya, kaltsiya, kobalta, kremniya, litiya, magniya, margantsa, medi, molibdena, myshyaka, natriya, nikelya, olova, svintsa, selena, serebra, strontsiya, surmy, talliya, tellura, titana, khroma, tsinka $v$ pitevykh, prirodnykh i stochnykh vodakh na atomno-absorbtsionnom spektrometre «KVANT.Z» s elektrotermicheskoy atomizatsiey [Methodological Instructions for Measuring the Mass Concentrations of Aluminum, Barium, Beryllium, Vanadium, Bismuth, Iron, Cadmium, Potassium, Calcium, Cobalt, Silicon, Lithium, Magnesium, Manganese, Copper, Molybdenum, Arsenic, Sodium, Nickel, Tin, Lead, Selenium, Silver, Strontium, Antimony, Thallium, Tellurium, Titanium, Chromium, Zinc in Drinking, Natural and Waste Waters on the Kvant.Z Atomic Absorption Spectrometer with Electrothermal Atomization]. Moscow, 2010. 8 p.

9. Metodicheskie ukazaniya po opredeleniyu tyazhelykh metallov $v$ pochvakh selkhozugodiy $i$ produktsii rastenievodstva [Methodological Guidelines for the Determination of Heavy Metals in Soils of Agricultural Lands and Crop Production]. Moscow, Tayfun Publ., 1992. 31 p.

10. MUK 4.1.1471-03. Atomno-absorbtsionnoe opredelenie massovoy kontsentratsii rtuti v pochvakh $i$ tverdykh mineralnykh materialakh: (utv. Minzdravom Rossii 29.06.2003) [MUK 4.1.1471-03. Atomic Absorption Determination of the Mass Concentration of Mercury in Soils and Hard Mineral Materials: (approved by the Ministry of Health of Russia on June 29, 2003)]. Moscow, 2003. 10 p.

11. O predpriyatii VMK «Krasnyy Oktyabr» [About the Krasnyy Oktyabr Enterprise]. URL: http:// www.vmzko.ru/home/about.html. (accessed February 15, 2017).

12. RD 52.18.191-89. Metodicheskie ukazaniya. Metodika vypolneniya izmereniy massovoy doli kislotorastvorimykh form metallov (medi, svintsa, tsinka, nikelya, kadmiya) $v$ probakh pochvy atomnoabsorbtsionnym analizom: (utv. Goskomgidrometom SSSR) [RD 52.18.191-89. Methodical Instructions. Method for Performing Measurements of the Mass Fraction of Acid-Soluble Forms of Metals (Copper, Lead, Zinc, Nickel, Cadmium) in Soil Samples by Atomic Absorption Analysis (Approved by the State Committee for the Hydrometeorology of the USSR)]. Moscow, 1990. 37 p.
13. Spektrometr atomno-absorbtsionnyy $s$ elektrotermicheskoy atomizatsiey «KVANT.Z» [Atomic Absorption Spectrometer with Electrothermal Atomization KVANT.Z]. URL: http://www.cortec.ru/ index.php?id=30. (accessed February 15, 2017).

14. Safarova V.I., Shaydulina G.F., Mikheeva T.N. Sposoby probopodgotovki pochvy, donnykh otlozheniy i tverdykh otkhodov dlya atomnoabsorbtsionnogo opredeleniya tyazhelykh metallov [Methods of Sample Preparation of Soil, Bottom Sediments and Solid Waste for Atomic Absorption of Heavy Metals]. Zavodskaya laboratoriya. Diagnostika materialov, 2010, vol. 76, no. 2, pp. 10-14.

15. Atomabsorptionsspektrometer - Aufbau und virtuelles Messgerät. ChemgaPedia. URL: http:// www.chemgapedia.de/vsengine/vlu/vsc/de/ch/16/ anac/aasvirtmess.vlu/Page/vsc/de/ch/16/anac/ aas5_unt1.vscml.html. (accessed February 15, 2017).

16. Atomabsorptionsspektroskopie (AAS) Anwendung. ChemgaPedia 14.02.2017. URL: http:// www.chemgapedia.de/vsengine/vlu/vsc/de/ch/16/ anac/aasanwend.vlu.html. (accessed February 14, 2017).

17. Atomabsorptionsspektroskopie (AAS) und Atomemissionsspektroskopie (AES). Johannes Gutenberg-Universität Mainz 16.02.2017. URL: http:// www.ak-hoffmann.chemie.uni-mainz.de/pdf/script/ AnalytC-Teil-4-folien.pdf.

18. Atomabsorptionsspektrometrie (AAS). Universität Regensburg. URL: http:/www-app.uniregensburg.de/Fakultaeten/CHP/Analytische Chemie/web/dateien/duerkop/AAS.pdf. (accessed February 16, 2017).

19. Atomic absorption spectrometers (AAS). Green Lab Hungary Engineering, Ltd16.02.2017. URL: http://www.greenlab.hu/atomic-absorptionspectrometers-aas4.html?tid=zeenit-atomicabsorption-spectrometer-aas.

20. Einführung in dieAtomabsorptionsspektroskopie (AAS). ChemgaPedia. URL: http://www.chemgapedia.de/ vsengine/vlu/vsc/de/ch/16/ anac/aaseinf.vlu.html. (accessed February 14, 2017).

21. Klemm K., Özmen B. Schnell! Einfach! Wirtschaftlich! Schnelle Multielement-Analyse von Schwermetallen in Boden- und Sedimentproben mit dem contrAA®. Analytik Jena. 16.02.2017. URL: https://www.analytik-jena.de/en/analyticalinstrumentation/ssc/applicationsupport/downloads. html?tx padb_dam $\% 5$ Bpublication $\% 5 \mathrm{D}=\% 0 \mathrm{~A} \% \mathrm{E}$ E\%02y\%FCM $\%$ C6\%D8\&tx_padb_dam\%5Baction $\% 5$ $\mathrm{D}=$ download\&tx_padb_dam $\% 5 \mathrm{~B}$ controller $\% 5 \mathrm{D}=$ Download $\& \mathrm{cHash}=\mathrm{a} 3 \mathrm{e} 4 \mathrm{de} 429 \mathrm{~d} 82 \mathrm{a} 36 \mathrm{bb} 83 \mathrm{c} 8 \mathrm{~b} 19 \mathrm{e} 159 \mathrm{~b} 633$.

22. Schwermetall-Emissionen. Das Umweltbundesamt. URL: http://www.umweltbundesamt.de/daten/ luftbelastung/luftschadstoff-emissionen-indeutschland/schwermetall-emissionen\#textpart-1. (accessed February 14, 2017). 
A.А. Тихонова. Атомно-абсорбционная спектрометрия

23. Spangl W., Nagl C. Jahresbericht der Luftgütemessungen in Österreich 2015. Wien, Umweltbundesamt GmbH, 2016. 152 p.
24. Spektrallinien. ChemgaPedia. URL: http://www. chemgapedia.de/vsengine/vlu/vsc/de/ch/13/vlu/analytik/ aas/spektrallinien.vlu.html. (accessed February 14, 2017).

\section{Information about the Author}

Anna A. Tikhonova, Postgraduate Student, Department of Ecology and Nature Management, Volgograd State University, Prosp. Universitetsky, 100, 400062 Volgograd, Russian Federation, tihonova@volsu.ru.

\section{Информация об авторе}

Анна Афанасьевна Тихонова, аспирант кафедры экологии и природопользования, Волгоградский государственный университет, просп. Университетский, 100, 400062 г. Волгоград, Российская Федерация, tihonova@volsu.ru. 